\title{
THE HYPERELLIPTIC MAPPING CLASS GROUP OF KLEIN SURFACES
}

\author{
E. BUJALANCE ${ }^{1}$, A. F. COSTA ${ }^{1}$ AND J. M. GAMBOA ${ }^{2}$ \\ ${ }^{1}$ Departamento de Matemáticas Fundamentales, Facultad de Ciencias, \\ Universidad Nacional de Educación a Distancia, \\ Senda del Rey, 28040 Madrid, Spain \\ ${ }^{2}$ Departamento de Algebra, Facultad de Ciencias Matemáticas, \\ Universidad Complutense, 28040 Madrid, Spain
}

(Received 24 March 1999)

\begin{abstract}
In this paper we study the algebraic structure of the hyperelliptic mapping class group of Klein surfaces, which is closely related to the mapping class group of punctured discs. This group plays an important role in the study of the moduli space of hyperelliptic real algebraic curves. Our main result provides a presentation by generators and relations for the hyperelliptic mapping class group of surfaces of prescribed topological type.
\end{abstract}

Keywords: Klein surface; hyperelliptic Klein surface; mapping class group; real algebraic curve; moduli space

AMS 2000 Mathematics subject classification: Primary 14H10; 20H10; 30F50

\section{Introduction}

The study of the mapping class group goes back to the classical works of Fricke, Artin, Magnus, Bohnenblust, Markoff and others. Most of this work concerns the braid group and the mapping class group of punctured spheres. This group has been extremely useful in understanding the moduli space of hyperelliptic complex algebraic curves because it is intimately related with the hyperelliptic mapping class group of the hyperelliptic Riemann surfaces by virtue of the papers by Birman and Hilden [4], Harvey and Maclachlan [7] and Zieschang [13]. Our goal in this paper is to study the algebraic structure of the hyperelliptic mapping class group of Klein surfaces, which, as we will see, is closely related to the mapping class group of punctured discs. This group plays an important role, analogous to the one in the complex case, in the study of the moduli space of hyperelliptic real algebraic curves.

Our main result provides a presentation by generators and relations for the hyperelliptic mapping class group of surfaces of prescribed topological type (for the mapping class group of Riemann surfaces, such a presentation was obtained in [8]).

The article is divided into the following sections. Section 2 is devoted to introducing the notions and notation we are going to use. In $\S 3$ we establish the relationship between the 
subset of the moduli space, determined by the hyperelliptic Klein surfaces of prescribed topological type, and its hyperelliptic mapping class group. In $\S 4$ we get a presentation of the modular group of the group uniformizing the quotient of the Klein surface under its hyperelliptic involution. This is useful to get a presentation, in $\S 5$, of the relative modular group or hyperelliptic mapping class group of hyperelliptic Klein surfaces. Finally, we obtain some applications in $\S 6$; in particular we determine under what conditions the hyperelliptic mapping class group is finite.

\section{Preliminaries}

A Klein surface is a surface (orientable or not, with or without boundary) endowed with a dianalytic structure. Let $X$ be a Klein surface of algebraic genus $p \geqslant 2$ and non-empty boundary. The surface $X$ is said to be hyperelliptic if it admits a dianalytic involution $\phi$ such that the quotient $X /\langle\phi\rangle$ has algebraic genus zero. It is well known that such an involution is unique, and it is called the hyperelliptic involution.

Notice that there exists a functorial equivalence between compact hyperelliptic Klein surfaces and hyperelliptic real algebraic curves (see $[\mathbf{1}, \mathbf{5}]$ ) analogous to the classical one, and so we can express the obtained results in terms of hyperelliptic real algebraic curves.

The surface $X$ can be written as a quotient $X=U / \Gamma$ of the hyperbolic plane $U$ under the action of a surface non-Euclidean crystallographic (NEC) group $\Gamma$. The hyperellipticity of $X$ means that the quotient $X /\langle\phi\rangle$ can be uniformized by an NEC group $\Gamma^{\prime}$ containing $\Gamma$ as a subgroup of index 2, i.e. $X /\langle\phi\rangle=U / \Gamma^{\prime}$.

The algebraic presentation of the group $\Gamma^{\prime}$-or, in other words, the topological data of the covering $X \rightarrow X /\langle\phi\rangle$-is determined by a symbol, the signature of $\Gamma^{\prime}$, which has the following form:

$$
\sigma\left(\Gamma^{\prime}\right)=(0 ;+;[2, . r ., 2] ;\{(2, . \stackrel{s}{.}, 2)\}),
$$

where $r$ and $s$ are non-negative integers, and $s$ is even. That means that $X /\langle\phi\rangle$ is a topological disc and the covering $X \rightarrow X /\langle\phi\rangle$ ramifies at $r$ inner points of $X /\langle\phi\rangle$ and $s$ points in the boundary. In other words, $X /\langle\phi\rangle$ has an orbifold structure (see [12]) with $r$ conic points and $s$ corner points, and $\Gamma^{\prime}$ can be considered as the fundamental group of the orbifold $X /\langle\phi\rangle$.

Let $g$ be the topological genus of $X, k$ the number of connected components of its boundary, and $\varepsilon=2$ if $X$ is orientable and $\varepsilon=1$ otherwise. We call the triple $(g, k, \varepsilon)$ the topological type of $X$, which determines the integers $r, s$ in (2.1). In fact, it is known (see $[\mathbf{5}$, ch. 2]) that

$$
\left.\begin{array}{ll}
\text { if } g=0, & \text { then } r=0 \text { and } s=2 k ; \\
\text { if } g \neq 0 \text { and } \varepsilon=2(\text { and so } k<3), & \text { then } r=2 g+k, s=0 ; \\
\text { if } g \neq 0 \text { and } \varepsilon=1, & \text { then } r=g \text { and } s=2 k .
\end{array}\right\}
$$

Note that we always assume that the algebraic genus $p=\varepsilon g+k-1$ of $X$ is greater than or equal to 2 .

For all these general results concerning hyperelliptic Klein surfaces, the interested reader is referred to $[\mathbf{5}]$. 
In classical terms, the mapping class group of an orientable topological surface without boundary is defined as the group of isotopy classes of orientation-preserving autohomeomorphisms of the surface. We define the mapping class group of the Klein surface $X$ as the group of isotopy classes of autohomeomorphisms of the underlying topological surface. This is isomorphic to the so-called modular group of $\Gamma$, defined as

$$
\bmod (\Gamma)=\frac{\operatorname{aut}(\Gamma)}{\operatorname{inn}(\Gamma)}
$$

where $\operatorname{aut}(\Gamma)$ is the group of automorphisms of $\Gamma$ and $\operatorname{inn}(\Gamma)$ is the subgroup consisting of its inner automorphisms (see [13, Corollary 8.8]).

If $X$ is hyperelliptic, the group

$$
\bmod \left(\Gamma^{\prime}\right)=\frac{\operatorname{aut}\left(\Gamma^{\prime}\right)}{\operatorname{inn}\left(\Gamma^{\prime}\right)}
$$

is called the modular group of $\Gamma^{\prime}$. Since $\Gamma$ is a normal subgroup of $\Gamma^{\prime}$, the group aut $\left(\Gamma^{\prime}, \Gamma\right)$, automorphisms $f$ of $\Gamma^{\prime}$ such that $f(\Gamma)=\Gamma$, contains inn $\left(\Gamma^{\prime}\right)$. The quotient

$$
\bmod \left(\Gamma^{\prime}, \Gamma\right)=\frac{\operatorname{aut}\left(\Gamma^{\prime}, \Gamma\right)}{\operatorname{inn}\left(\Gamma^{\prime}\right)}
$$

is called the relative modular group, or the hyperelliptic mapping class group of fixed topological type $(g, k, \varepsilon)$, and we shall denote it by $\operatorname{map}^{h}(g, k, \varepsilon)$. The group map ${ }^{h}(g, k, \varepsilon)$ is isomorphic to the mapping class group of the orbifold $X /\langle\phi\rangle$, i.e. the group of isotopy classes of homeomorphisms preserving the singular points and its type. This approach will be used in $\S 4$.

\section{Moduli of hyperelliptic Klein surfaces}

We are mainly interested in determining the group $\operatorname{map}^{h}(g, k, \varepsilon)$, since it plays a central role in describing the moduli space of hyperelliptic Klein surfaces. Let us determine the relation between these two objects, but first we introduce some more notation. Let $G$ be the group of maps from $\hat{\boldsymbol{C}}=\boldsymbol{C} \cup\{\infty\}$ to $\hat{\boldsymbol{C}}$ having the form

$$
z \mapsto \frac{a z+b}{c z+d} \quad \text { or } \quad z \mapsto \frac{a^{\prime} \bar{z}+b^{\prime}}{c^{\prime} \bar{z}+d^{\prime}}
$$

where $\bar{z}$ is the complex conjugate of $z ; a, b, c, d, a^{\prime}, b^{\prime}, c^{\prime}$ and $d^{\prime}$ are real numbers;

$$
\operatorname{det}\left(\begin{array}{ll}
a & b \\
c & d
\end{array}\right)=1 \quad \text { and } \operatorname{det}\left(\begin{array}{ll}
a^{\prime} & b^{\prime} \\
c^{\prime} & d^{\prime}
\end{array}\right)=-1
$$

For each NEC group $\Delta$, let $M(\Delta, G)$ be the set of type-preserving group monomorphisms $\Delta \hookrightarrow G$ with discrete image (see [7] for the analogous notion for Fuchsian groups). The Teichmüller space of $\Delta$ is the quotient

$$
T(\Delta)=\frac{M(\Delta, G)}{\sim}
$$


where $\sim$ is the equivalence relation given by conjugation by elements of $G$. In particular, the inclusion $i: \Gamma \hookrightarrow \Gamma^{\prime}$ between the NEC groups $\Gamma$ and $\Gamma^{\prime}$ uniformizing $X$ and $X /\langle\phi\rangle$, induces a map

$$
i_{*}: M\left(\Gamma^{\prime}, G\right) \rightarrow M(\Gamma, G): \alpha \longmapsto \alpha \circ i,
$$

which is compatible with the equivalence relation $\sim$, and so one gets a map

$$
m: T\left(\Gamma^{\prime}\right) \longrightarrow T(\Gamma):[\alpha] \longmapsto[\alpha \circ i],
$$

which is an isometric embedding with respect to the Teichmüller metric (see [10]).

The group aut $(\Gamma)$ acts in a natural way on $T(\Gamma)$, and this action induces the following map:

$$
\bmod (\Gamma) \times T(\Gamma) \rightarrow T(\Gamma):([\varphi],[\alpha]) \longmapsto[\alpha \circ \varphi] .
$$

Then, as in the case of Riemann surfaces (see $[\mathbf{7}, \S 4]$ ), the moduli space of hyperelliptic Klein surfaces of topological type $(g, k, \varepsilon)$ is defined as

$$
M_{h}(g, k, \varepsilon)=\Lambda(\Gamma) / \bmod (\Gamma)
$$

where

$$
\Lambda(\Gamma)=\bigcup_{[\varphi] \in \bmod (\Gamma)}[\varphi]\left(m\left(T\left(\Gamma^{\prime}\right)\right)\right) .
$$

On the other hand, the action of $\bmod \left(\Gamma^{\prime}\right)$ on $T\left(\Gamma^{\prime}\right)$ restricts to an action of $\bmod \left(\Gamma^{\prime}, \Gamma\right)$ on $T\left(\Gamma^{\prime}\right)$, and so the quotient $T\left(\Gamma^{\prime}\right) / \bmod \left(\Gamma^{\prime}, \Gamma\right)$ makes sense. We are now in a position to prove the main result of this section.

Theorem 3.1. There exists a homeomorphism between the moduli space $M_{h}(g, k, \varepsilon)$ and the quotient $T\left(\Gamma^{\prime}\right) / \bmod \left(\Gamma^{\prime}, \Gamma\right)$.

So, as in the classical case of Riemann surfaces, the relative modular group $\bmod \left(\Gamma^{\prime}, \Gamma\right)$ or the hyperelliptic mapping class group $\operatorname{map}^{h}(g, k, \varepsilon)$ allows us to present the moduli space of hyperelliptic Klein surfaces of given topological type as a quotient of a Teichmüller space.

Proof of Theorem 3.1. The map $\phi: T\left(\Gamma^{\prime}\right) \rightarrow M_{h}(g, k, \varepsilon)$ defined as $\phi([\alpha])=$ $[m([\alpha])]_{\bmod (\Gamma)}$ is obviously surjective. Moreover, if $\phi([\alpha])=\phi([\beta])$ for some $[\alpha]$ and $[\beta]$ in $T\left(\Gamma^{\prime}\right)$, then $[\beta \circ i]=[\alpha \circ i \circ \varphi]$ for some $\varphi \in \operatorname{aut}(\Gamma)$, and so there exists $g \in G$ such that $\alpha \circ i \circ \varphi=c_{g} \circ \beta \circ i$, where $c_{g}$ denotes conjugation by $g$. Hence, if $j=c_{g} \circ \beta$, then $\alpha(i(\Gamma))=$ $j(i(\Gamma))$. This implies, by the uniqueness of the hyperelliptic involution, that the groups $\alpha\left(\Gamma^{\prime}\right)$ and $j\left(\Gamma^{\prime}\right)$ coincide, since both are isomorphic to $\Gamma^{\prime}$ and contain $\alpha(i(\Gamma))=j(i(\Gamma))$ as a subgroup of index 2. Thus $\psi=\alpha^{-1} \circ j \in \operatorname{aut}\left(\Gamma^{\prime}, \Gamma\right)$ and $[\alpha \circ \psi]=[j]=[\beta]$.

From the above and Corollary 8.9 and Theorem 9.12 of [10], it follows that the map

$$
\hat{\phi}: \frac{T\left(\Gamma^{\prime}\right)}{\bmod \left(\Gamma^{\prime}, \Gamma\right)} \longrightarrow M_{h}(g, k, \varepsilon),
$$

induced by $\phi$, is a homeomorphism. 


\section{The modular group $\bmod \left(\Gamma^{\prime}\right)$}

As we have already said, our goal in this paper is to compute the group $\bmod \left(\Gamma^{\prime}, \Gamma\right)$. This will be done in the next section and, as a first step in this direction, we now compute $\bmod \left(\Gamma^{\prime}\right)$.

Let $D$ be the closed unit disc, and let us fix two sets, $A_{1} \subset D^{\circ}=$ interior of $D$ and $A_{2} \subset \partial D$, of cardinality $r$ and $s$, respectively. Obviously, the set $\Sigma=\Sigma_{r, s}$ of autohomeomorphisms $\varphi$ of $D$, such that $\varphi\left(A_{1}\right)=A_{1}$ and $\varphi\left(A_{2}\right)=A_{2}$, is a group under composition. Two elements $\varphi$ and $\psi$ in $\Sigma$ are said to be equivalent, and denoted $\varphi \sim \psi$, if there exists a homotopy

$$
H: I \times D \rightarrow D,
$$

where $I$ is the closed interval $[0,1]$, such that $H_{0}=\varphi, H_{1}=\psi$, and for each $t \in I$ :

$$
H_{t}(\partial D)=\partial D, \quad H_{t}\left(A_{1}\right)=A_{1} \quad \text { and } \quad H_{t}\left(A_{2}\right)=A_{2} .
$$

The quotient $\Sigma / \sim$ is the modular $\operatorname{group} \bmod _{r, s}(D)$ of the punctured disc, and the following proposition is an easy consequence of the results of Zieschang [13].

Proposition 4.1. The groups $\bmod _{r, s}(D)$ and $\bmod \left(\Gamma^{\prime}\right)$ are isomorphic.

For every $s \geqslant 2$, let us label $A_{2}=\left\{b_{0}, \ldots, b_{s-1}\right\}$, and let $G_{s}$ be the group of contiguitypreserving permutations of $A_{2}$ (of course, $b_{0}$ and $b_{s-1}$ are contiguous). Clearly, $G_{2}$ is the cyclic group of order 2 generated by $\sigma: b_{i} \longmapsto b_{1-i}$, and for $s>2, G_{s}$ is the dihedral group $D_{s}$ of order $2 s$ generated by the rotation $\rho: b_{i} \longmapsto b_{i-1}, 1 \leqslant i \leqslant s-1 ; b_{0} \longmapsto b_{s-1}$, and the symmetry $\sigma: b_{i} \longmapsto b_{s-i-1}, 0 \leqslant i \leqslant s-1$.

From Macbeath [9], the restriction $\varphi \mid A_{2}$ of each $\varphi \in \Sigma$ occurs in $G_{s}$, and in fact one gets a group epimorphism

$$
\pi: \bmod _{r, s}(D) \rightarrow G_{s}:[\varphi] \longmapsto \varphi \mid A_{2} .
$$

To see this, we only need to observe that for a homotopy $H: I \times D \longrightarrow D$ with $H_{0}=\varphi$ and $H_{1}=\psi$, and every point $x \in A_{2}$, the image $H(I \times\{x\})$ is a connected subset of $A_{2}$, i.e. a unique point $y_{x} \in A_{2}$, and so $\varphi(x)=H(0, x)=y_{x}=H(1, x)=\psi(x)$. Consequently, $G_{s}$ is isomorphic to the quotient $\bmod _{r, s}(G) / \operatorname{ker} \pi$.

Proposition 4.2. If $s \geqslant 2$, the $\operatorname{group} \bmod \left(\Gamma^{\prime}\right)=\bmod _{r, s}(D)$ is a semidirect product $G_{s} \propto \operatorname{ker} \pi$.

Proof. In order to show that $\bmod _{r, s}(D)$ is a semidirect product of $G_{s}$ and $\operatorname{ker} \pi$ it suffices to define a section $\varphi: G_{s} \rightarrow \bmod _{r, s}(D)$ of $\pi$. Since $\rho$ and $\sigma$ are the generators of $G_{s}$ (or just $\sigma$ if $s=2$ ) it is enough to construct $\varphi(\rho)=\hat{\rho}$ and $\varphi(\sigma)=\hat{\sigma}$. In fact, using the isomorphism between $\bmod _{r, s}(D)$ and $\bmod \left(\Gamma^{\prime}\right)$, we shall first construct $\hat{\rho}$ and $\hat{\sigma}$ as elements in $\bmod \left(\Gamma^{\prime}\right)$. For this purpose, let us denote by

$$
\left\{x_{1}, \ldots, x_{r}, e, c_{0}, \ldots, c_{s}\right\}
$$




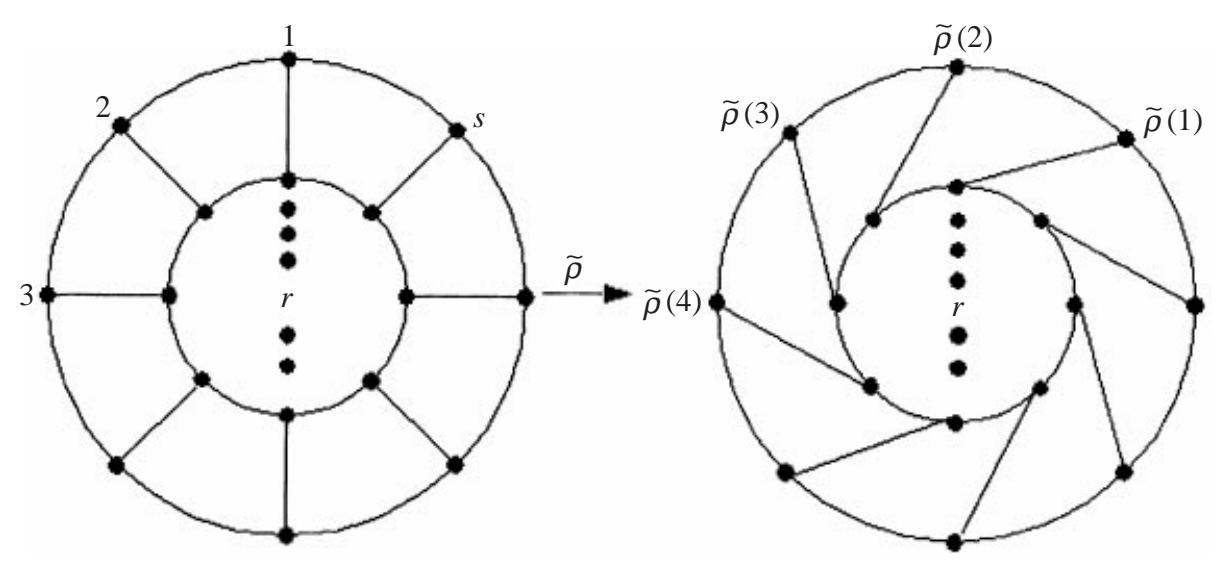

Figure 1. The automorphism $\tilde{\rho}$.

a set of canonical generators of $\Gamma^{\prime}$, where each $x_{i}$ is elliptic of order $2, e$ is hyperbolic, each $c_{j}$ is a reflection and

$$
\left(c_{j} c_{j+1}\right)^{2}=1 ; x_{1} \ldots x_{r} . e=1 ; e^{-1} c_{0} e c_{s}=1 .
$$

We fix this notation throughout the paper.

Let us consider the automorphisms of $\Gamma^{\prime}$ induced by the assignments:

$$
\left.\begin{array}{c}
\hat{\rho}: x_{i} \mapsto x_{i} ; \quad e \mapsto e ; \quad c_{0} \mapsto e c_{s-1} e^{-1} ; \quad c_{j} \mapsto c_{j-1}, \quad 1 \leqslant j \leqslant s ; \\
\hat{\sigma}: x_{1} \mapsto x_{1} ; \quad x_{i} \mapsto y_{i} x_{i} y_{i}^{-1}, \quad 2 \leqslant i \leqslant r ; \quad e \mapsto e^{-1} ; \quad c_{0} \mapsto e^{-1} c_{1} e ; \\
c_{j} \mapsto c_{s-j+1}, \quad 1 \leqslant j \leqslant s,
\end{array}\right\}
$$

where $y_{i}=x_{1} \ldots x_{i-1}$ for $2 \leqslant i \leqslant r$.

Given the point $b_{j+1} \in A_{2}$ as the intersection of the axes of the reflections $c_{j}$ and $c_{j+1}$, then $\pi(\hat{\rho})\left(b_{j+1}\right)$ is the intersection of the axes of $\hat{\rho}\left(c_{j}\right)=c_{j-1}$ and $\hat{\rho}\left(c_{j+1}\right)=c_{j}$, i.e

$$
\pi([\hat{\rho}])\left(b_{j+1}\right)=b_{j}=\rho\left(b_{j}\right),
$$

and so $\pi([\hat{\rho}])=\rho$. Analogously $\pi([\hat{\sigma}])=\sigma$, and we say that $[\hat{\rho}]$ and $[\hat{\sigma}]$ represent $\rho$ and $\sigma$, respectively. Note that $[\hat{\rho}]^{s}=[\hat{\sigma}]^{2}=1$.

The classes $[\hat{\rho}],[\hat{\sigma}]$ can be represented as the automorphisms $\tilde{\rho}, \tilde{\sigma}$ of the orbifold $X /\langle\phi\rangle$ shown in Figures 1 and 2. The automorphisms given by $\tilde{\rho}$ and $\tilde{\sigma}$ in the orbifold fundamental group of $X /\langle\phi\rangle$ are exactly $\hat{\rho}$ and $\hat{\sigma}$.

We shall now obtain a presentation by generators and relations of $\bmod _{r, s}(D)$. For this purpose we compute ker $\pi$ and we study first the case $s>2$. Then, the elements in ker $\pi$ are represented by homeomorphisms of $D$ (which leave the boundary $\partial D$ invariant), and fix $s \geqslant 4$ points in $\partial D$, and so all of them preserve the orientation. Let $\Delta=$ $D \backslash A_{1}$ and $F_{r}$ be the free group generated by $X_{1}, \ldots, X_{r}$. The braid group $B_{r}$ of the plane $E^{2}$ is the subgroup of the automorphism group aut $\left(F_{r}\right)$ of $F_{r}$ consisting of those 


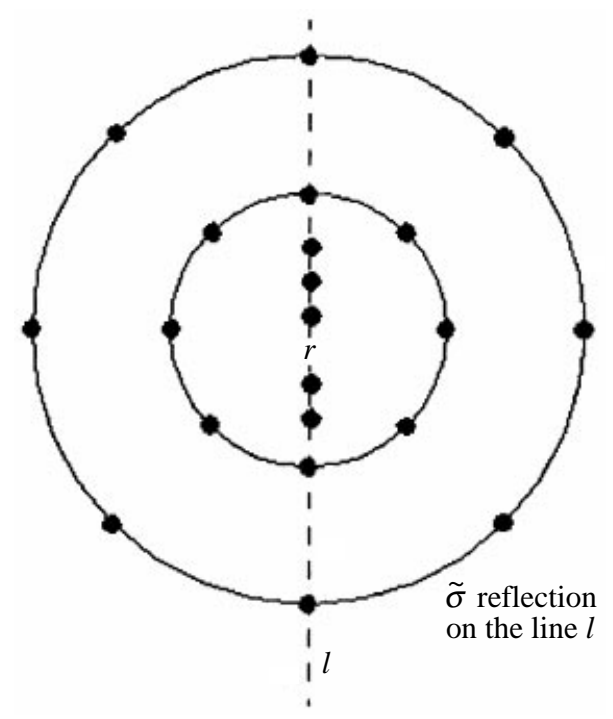

Figure 2. The automorphism $\tilde{\sigma}$.

automorphisms of $F_{r}$ which fix the product $X_{1} \ldots X_{r}$ and map each generator $X_{i}$ to a conjugate of some generator $X_{j}$. If we denote by $I\left(B_{r}\right)=B_{r} \cap \operatorname{inn}\left(F_{r}\right)$ the intersection of $B_{r}$ with the subgroup of inner automorphisms of $F_{r}$, Birman [2] proved the existence of an epimorphism

$$
\theta: \operatorname{homeo}(\Delta ; \partial \Delta) \longrightarrow \frac{B_{r}}{I\left(B_{r}\right)}
$$

from the group homeo $(\Delta ; \partial \Delta)$ of homeomorphisms of $\Delta$ leaving $\partial \Delta$ invariant, onto the quotient $B_{r} / I\left(B_{r}\right)$, whose kernel consists of those homeomorphisms homotopic to the identity. Thus, since

$$
\operatorname{ker} \pi \approx \frac{\operatorname{homeo}(\Delta ; \partial \Delta)}{\operatorname{ker} \theta} \approx \frac{B_{r}}{I\left(B_{r}\right)},
$$

it is enough to describe this last quotient. With the notation in Magnus [11]:

$$
\begin{gathered}
B_{r}=\left\langle\delta_{1}, \ldots, \delta_{r-1}: \delta_{i} \delta_{j}=\delta_{j} \delta_{i} \text { if }|i-j| \geqslant 2 ; \delta_{i} \delta_{i+1} \delta_{i}=\delta_{i+1} \delta_{i} \delta_{i+1} \text { and } 1 \leqslant i \leqslant r-2\right\rangle ; \\
A_{r}^{*}=\frac{B_{r}}{I\left(B_{r}\right)}=\left\langle\delta_{1}, \ldots, \delta_{r-1}: \delta_{i} \delta_{j}=\delta_{j} \delta_{i} \text { if }|i-j| \geqslant 2 ;\right. \\
\left.\delta_{i} \delta_{i+1} \delta_{i}=\delta_{i+1} \delta_{i} \delta_{i+1}, 1 \leqslant i \leqslant r-2 ;\left(\delta_{1}, \ldots, \delta_{r-1}\right)^{r}=1\right\rangle,
\end{gathered}
$$

where $A_{0}^{*}=A_{1}^{*}$ is the trivial group. Note also that $A_{2}^{*}=\boldsymbol{Z} / 2 \boldsymbol{Z}$.

By (4.1) we know how to represent the generators of $D_{s}$ as elements in $\bmod \left(\Gamma^{\prime}\right)$; to understand the meaning of the semidirect product $D_{s} \propto A_{r}^{*}$ we must provide an explicit presentation of the generators $\delta_{j}$ of $A_{r}^{*}$ as elements in $\bmod \left(\Gamma^{\prime}\right)$. Let $r \geqslant 2$, and for every $1 \leqslant j \leqslant r-1$, let $\alpha_{j}: \Gamma^{\prime} \longrightarrow \Gamma^{\prime}$ be the automorphism induced by the assignment

$$
\left.\begin{array}{rl}
x_{j} \mapsto x_{j+1} ; \quad x_{j+1} \mapsto x_{j+1} x_{j} x_{j+1} ; \quad x_{i} \longmapsto x_{i} \quad \text { if } j \neq i \neq j+1 ; \\
e \longmapsto e ; \quad c_{k} \longmapsto c_{k} \quad \text { for } 0 \leqslant k \leqslant s .
\end{array}\right\}
$$


It is easy to check that $\alpha_{j} \hat{\rho}=\hat{\rho} \alpha_{j}$ and $\alpha_{j} \hat{\sigma} \alpha_{j}=\hat{\sigma}$. Summarizing, and since $A_{r}^{*}$ is trivial if $r<2$, we can now give a presentation for $\bmod _{r, s}(D)$ if $s>2$.

\section{Theorem 4.3.}

(i) If $s>2$ and $r \geqslant 2$, then $\bmod _{r, s}(D)=D_{s} \propto A_{r}^{*}$, where

$$
\begin{aligned}
& D_{s} \propto A_{r}^{*}=\left\langle\delta_{1}, \ldots, \delta_{r-1}, \rho, \sigma: \rho^{s}=\sigma^{2}=1 ; \delta_{i} \delta_{j}=\delta_{j} \delta_{i} \text { if }|i-j| \geqslant 2 ;\right. \\
& \delta_{i} \delta_{i+1} \delta_{i}=\delta_{i+1} \delta_{i} \delta_{i+1}, 1 \leqslant i \leqslant r-2 ; \quad\left(\delta_{1} \ldots \delta_{r-1}\right)^{r}=1 ; \\
&\left.\delta_{j} \rho=\rho \delta_{j} ; \delta_{j} \sigma \delta_{j}=\sigma, 1 \leqslant i \leqslant r-1\right\rangle .
\end{aligned}
$$

(ii) If $s>2$ and $r<2$, then $\bmod _{r, s}(D)=D_{s}$.

Moreover, formulae (4.1) and (4.2) show the way the generators of $\bmod \left(\Gamma^{\prime}\right)$ can be expressed as (classes of) automorphisms of $\Gamma^{\prime}$.

The cases $s=2$ and $s=0$ follow similar arguments as the previous one. We just sketch the proof, pointing out the specific facts for these cases.

Assume now that $s=2$, and let us denote by $R$ the reflection of the disc $D$ whose axis is the line joining the two points in $A_{2}$. Each homeomorphism of $D$ whose restriction to $A_{2}$ is the identity is homotopic to either $R$ or the identity, and so we get an epimorphism,

$$
\eta: \operatorname{ker} \pi \longrightarrow \boldsymbol{Z} / 2 \boldsymbol{Z}:[\varphi] \longmapsto \begin{cases}1, & \text { if } \varphi \text { is homotopic to } R, \\ 0, & \text { otherwise, }\end{cases}
$$

whose kernel is $A_{r}^{*}$. Hence, $\operatorname{ker} \pi$ is a semidirect product $\operatorname{ker} \pi=\boldsymbol{Z} / 2 \boldsymbol{Z} \propto A_{r}^{*}$. Moreover, if $r \geqslant 2$, let $\hat{\mu}: \Gamma^{\prime} \longrightarrow \Gamma^{\prime}$ be the automorphism of $\Gamma^{\prime}$ induced by the assignment

$$
\left.\begin{array}{rl}
\hat{\mu}: x_{1} & \mapsto x_{1} ; \quad x_{i} \mapsto y_{i} x_{i} y_{i}^{-1}, \quad 2 \leqslant i ; \quad e \mapsto e^{-1} ; \\
c_{0} \mapsto e^{-1} c_{1} e ; \quad c_{k} \mapsto c_{3-k}, \quad k=1,2,
\end{array}\right\}
$$

where $y_{i}=x_{1} \ldots x_{i-1}$.

It is boring, but straightforward, to check that $\hat{\mu}$ is actually an automorphism of $\Gamma^{\prime}$ of order 2 such that $\alpha_{j} \hat{\mu} \alpha_{j}=\hat{\mu}, 1 \leqslant j \leqslant r-1$, where $\alpha_{1}, \ldots, \alpha_{r-1}$ are the automorphisms of $\Gamma^{\prime}$ defined in (4.2). Moreover, $\eta([\widehat{\mu}])=1$.

Also, with the notation of $(4.1)$, one can check that $(\hat{\sigma} \hat{\mu})^{2}$ is an inner automorphism of $\Gamma^{\prime}$ (conjugation by $\left.x_{r} \cdot x_{r-1} \ldots x_{1}\right)$, and so, if $\mu$ is the class of $\hat{\mu}, \bmod \operatorname{inn}\left(\Gamma^{\prime}\right)$, we obtain that if $s=2$ and $r \geqslant 2$, then

$$
\begin{array}{r}
\operatorname{ker} \pi=\boldsymbol{Z} / 2 \boldsymbol{Z} \propto A_{r}^{*}=\left\langle\delta_{1}, \ldots, \delta_{r-1}, \mu: \mu^{2}=1, \delta_{i} \delta_{j}=\delta_{j} \delta_{i} \text { if }|i-j| \geqslant 2 ;\right. \\
\delta_{i} \delta_{i+1} \delta_{i}=\delta_{i+1} \delta_{i} \delta_{i+1}, \text { for } 1 \leqslant i \leqslant r-2 ; \\
\left.\left(\delta_{1} \ldots \delta_{r-1}\right)^{r}=1 ; \delta_{i} \mu \delta_{i}=\mu, 1 \leqslant i \leqslant r-1\right\rangle .
\end{array}
$$

Consequently, if $r \geqslant 2$,

$$
\bmod _{r, 2}(D)=G_{s} \propto \operatorname{ker} \pi=\boldsymbol{Z} / 2 \boldsymbol{Z} \propto \operatorname{ker} \pi=\boldsymbol{Z} / 2 \boldsymbol{Z} \propto\left(\boldsymbol{Z} / 2 \boldsymbol{Z} \propto A_{r}^{*}\right) .
$$




\section{Theorem 4.4.}

(i) If $s=2$ and $r \geqslant 2, \bmod _{r, 2}(D)=D_{2} \propto A_{r}^{*}$, where

$$
\begin{array}{r}
D_{2} \propto A_{r}^{*}=\left\langle\sigma, \mu, \delta_{1}, \ldots, \delta_{r-1}: \sigma^{2}=\mu^{2}=(\sigma \mu)^{2}=1, \delta_{i} \delta_{j}=\delta_{j} \delta_{i} \text { if }|i-j| \geqslant 2 ;\right. \\
\delta_{i} \delta_{i+1} \delta_{i}=\delta_{i+1} \delta_{i} \delta_{i+1}, \text { for } 1 \leqslant i \leqslant r-2 ;\left(\delta_{1} \ldots \delta_{r-1}\right)^{r}=1 ; \\
\left.\delta_{i} \mu \delta_{i}=\mu, \delta_{i} \sigma \delta_{i}=\sigma, \text { for } 1 \leqslant i \leqslant r-1\right\rangle .
\end{array}
$$

(ii) If $s=2$ and $r \leqslant 1, \bmod _{r, 2}(D)=D_{2}$.

To finish this section we compute $\bmod _{r, 0}(D)$. Each homotopy class in $\bmod _{r, 0}(D)$ contains a representative $\varphi$ with a fixed point $p \in \partial D=S^{1}$. Hence, if $\varphi^{*}$ denotes the automorphism of the fundamental group $\pi_{1}\left(S^{1}\right)=\boldsymbol{Z}$ with base point $p$ induced by the restriction $\varphi \mid S^{1}$, one gets an epimorphism

$$
\bmod _{r, 0}(D) \rightarrow \boldsymbol{Z} / 2 \boldsymbol{Z}=\operatorname{aut}\left(\pi_{1}\left(S^{1}\right)\right):[\varphi] \mapsto \varphi^{*},
$$

with kernel $A_{r}^{*}$ (this epimorphism can be described in the same way as $\eta$ in the case $s=2)$. Hence $\bmod _{r, 0}(D)=\boldsymbol{Z} / 2 \boldsymbol{Z} \propto A_{r}^{*}$, where the generator $\varsigma$ of $\boldsymbol{Z} / 2 \boldsymbol{Z}$ can be seen as the class, $\bmod \operatorname{inn}\left(\Gamma^{\prime}\right)$, of the automorphism $\hat{\varsigma}: \Gamma^{\prime} \rightarrow \Gamma^{\prime}$ induced by the assignment

$$
x_{1} \mapsto x_{1}, \quad x_{i} \mapsto y_{i} x_{i} y_{i}^{-1}, \quad \text { for } 2 \leqslant i \leqslant r ; \quad e \mapsto e^{-1} ; \quad c_{0} \mapsto e^{-1} c_{0} e .
$$

It is easy to check that $\delta_{j} \varsigma \delta_{j}=\varsigma$, and so we have the following theorem.

\section{Theorem 4.5.}

(i) If $s=0$ and $r \geqslant 2$, then

$$
\begin{aligned}
& \bmod _{r, 0}(D)=\boldsymbol{Z} / 2 \boldsymbol{Z} \propto A_{r}^{*} \\
&=\left\langle\varsigma, \delta_{1}, \ldots, \delta_{r-1}: \varsigma^{2}=1, \delta_{i} \delta_{j}=\delta_{j} \delta_{i} \text { if }|i-j| \geqslant 2 ;\right. \\
& \delta_{i} \delta_{i+1} \delta_{i}=\delta_{i+1} \delta_{i} \delta_{i+1}, \text { for all } 1 \leqslant i \leqslant r-2 ; \\
&\left.\quad\left(\delta_{1} \ldots \delta_{r-1}\right)^{r}=1 ; \delta_{i} \varsigma \delta_{i}=\varsigma, \text { for } 1 \leqslant i \leqslant r-1\right\rangle .
\end{aligned}
$$

(ii) If $s=0$ and $r<2$, then $\bmod _{r, 0}(D)=\boldsymbol{Z} / 2 \boldsymbol{Z}$.

\section{The hyperelliptic mapping class group $\operatorname{map}^{h}(g, k, \varepsilon)$}

We have introduced the mapping class group $\operatorname{map}^{h}(g, k, \varepsilon)$ of hyperelliptic Klein surfaces of fixed topological type $(g, k, \varepsilon)$. The group $\operatorname{map}^{h}(g, k, \varepsilon)$ is isomorphic to the relative modular group

$$
\bmod \left(\Gamma^{\prime}, \Gamma\right)=\frac{\operatorname{aut}\left(\Gamma^{\prime}, \Gamma\right)}{\operatorname{inn}\left(\Gamma^{\prime}\right)}
$$

which is a subgroup of the modular group

$$
\bmod \left(\Gamma^{\prime}\right)=\frac{\operatorname{aut}\left(\Gamma^{\prime}\right)}{\operatorname{inn}\left(\Gamma^{\prime}\right)}
$$

that we computed in the previous section. 
Let $p: \Gamma^{\prime} \rightarrow \boldsymbol{Z} / 2 \boldsymbol{Z}$ be an epimorphism with ker $p=\Gamma$. It is clear that an automorphism $f \in \operatorname{aut}\left(\Gamma^{\prime}\right)$ occurs in aut $\left(\Gamma^{\prime}, \Gamma\right)$ if and only if $p \circ f=p$. We use this to prove the following theorem.

Theorem 5.1.

(i) If $s=0$, then $\bmod \left(\Gamma^{\prime}, \Gamma\right)=\bmod \left(\Gamma^{\prime}\right)=\boldsymbol{Z} / 2 \boldsymbol{Z} \propto A_{r}^{*}$.

(ii) If $s=2$, then $\bmod \left(\Gamma^{\prime}, \Gamma\right)=A_{r}^{*}$.

(iii) If $s>2$, then $\bmod \left(\Gamma^{\prime}, \Gamma\right)=D_{s / 2} \propto A_{r}^{*}$.

Proof. (i) If $s=0$, the epimorphism $p$ is defined by

$$
p: c_{0} \mapsto 0 ; \quad x_{i} \mapsto 1 ; \quad 1 \leqslant i \leqslant r ; \quad e \mapsto \begin{cases}0, & \text { if } r \text { is even, } \\ 1, & \text { if } r \text { is odd }\end{cases}
$$

With the notation in (4.2) and (4.4) it is easy to check the equalities $p \circ \alpha_{j}=p$, $1 \leqslant j \leqslant r-1$ and $p \circ \hat{\varsigma}=p$. Thus, from Theorem 4.5 it follows that $\bmod \left(\Gamma^{\prime}, \Gamma\right)=$ $\bmod \left(\Gamma^{\prime}\right)=\boldsymbol{Z} / 2 \boldsymbol{Z} \propto A_{r}^{*}$.

(ii), (iii) Now let $s \neq 0$. Then $p: \Gamma^{\prime} \rightarrow \boldsymbol{Z} / 2 \boldsymbol{Z}$ is defined by

$$
\begin{gathered}
p: c_{j} \mapsto \begin{cases}1, & \text { if } j \text { is even, } \\
0, & \text { if } j \text { is odd, } \quad x_{i} \mapsto 1, \quad 1 \leqslant i \leqslant r ;\end{cases} \\
e \mapsto \begin{cases}0, & \text { if } r \text { is even, } \\
1, & \text { if } r \text { is odd. }\end{cases}
\end{gathered}
$$

With the notation of (4.1), (4.2) and (4.3), one easily checks that $p \circ \alpha_{j}=p, 1 \leqslant j \leqslant$ $r-1 ; p \circ \hat{\sigma}=p$; but $p \circ \hat{\rho} \neq p$, if $s>2$ and $p \circ \hat{\mu} \neq p ; p \circ \hat{\sigma} \neq p$ if $s=2$. Thus, if we write $\bmod \left(\Gamma^{\prime}\right)=D_{s} \propto A_{r}^{*}=(\boldsymbol{Z} / s \boldsymbol{Z} \propto \boldsymbol{Z} / 2 \boldsymbol{Z}) \propto A_{r}^{*}$, then $\bmod \left(\Gamma^{\prime}, \Gamma\right)=D_{s / 2} \propto A_{r}^{*}$ if $s>2$, and $\bmod \left(\Gamma^{\prime}, \Gamma\right)=A_{r}^{*}$ if $s=2$.

Remark 5.2. Of course, from the proof above the semidirect product $D_{s / 2} \propto A_{r}^{*}$ if $s>2$ is easily presented by generators and relations using the presentations given in theorem 4.3 .

\section{Some consequences}

(1) If $g=0$, then $r=0$ and $s=2 k>2$, and so the hyperelliptic mapping class group $\operatorname{map}^{h}(0, k, 2)=D_{k}$.

(2) If $g \leqslant 2$ and $\varepsilon=1$, then

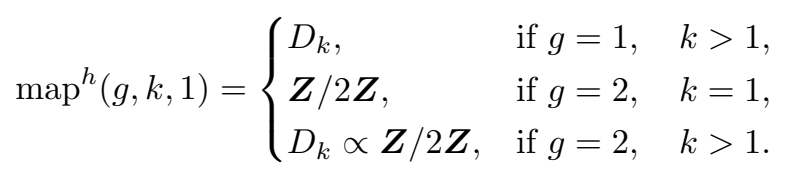


(3) Since $A_{r}^{*}$ is not finite for $r \geqslant 3$ and we just consider Klein surfaces of algebraic genus $p \geqslant 2$, i.e. $\varepsilon g+k \geqslant 3$, the cases quoted in (1) and (2) are the only ones in which the hyperelliptic mapping class group $\operatorname{map}^{h}(g, k, \varepsilon)$ is finite.

(4) In particular, since every Klein surface of algebraic genus $p=2$ is hyperelliptic, the mapping class groups

$$
\operatorname{map}(g, k, \varepsilon) \simeq \operatorname{map}^{h}(g, k, \varepsilon) \times \boldsymbol{Z} / 2 \boldsymbol{Z}
$$

if $\varepsilon g+k=3$, and since $k>0$, from the precedent analysis, it follows that they are finite unless $g=k=1, \varepsilon=2$.

(5) Birman and Chillingworth [3] studied the homeotopy group $H(X)$ of a non-orientable surface $X$, i.e. the quotient $H(X)=G(X) / D(X)$ of the group $G(X)$ of all autohomeomorphisms of $X$ under the subgroup $D(X)$ of homeomorphisms isotopic to the identity. In particular, they proved the following theorem (see [3, Theorem 3].

Theorem 6.1 (Birman-Chillingworth). Let $X$ be the sum of three projective planes. The homeotopy group $H(X)$ of $X$ has the following presentation:

$$
H(X)=\left\langle A, B, Y: A B A=B A B ; Y A Y^{-1}=A^{-1} ; Y B Y^{-1}=B^{-1} ; Y^{2}=(A B A)^{4}=1\right\rangle .
$$

To finish the paper we give another proof of this result with some extra information.

First of all, notice that $h=(A B A)^{2}$ has order 2 and it is easy to check that it is a central element of $H(X)$. Let $a, b, y$ be the classes of $A, B, Y \bmod \langle h\rangle$. Then $(a b)^{3}=a b a b a b=(a b a)^{2}=1$, and so

$$
H(X)=\langle h\rangle \times G(X)
$$

where

$$
G(X)=\left\langle a, b, y: a b a=b a b ; a y a=y ; b y b=y ; y^{2}=1,(a b)^{3}=1\right\rangle .
$$

On the other hand, the NEC group $\Gamma$ uniformizing $X$ admits a set $\left\{z_{1}, z_{2}, z_{3}\right\}$ of canonical generations which are glide reflections satisfying the relation $z_{1}^{2} z_{2}^{2} z_{3}^{2}=1$, and

$$
H(X)=\bmod (\Gamma)=\frac{\operatorname{aut}(\Gamma)}{\operatorname{inn}(\Gamma)} .
$$

In this case $g=3, k=0, \varepsilon=1$, and so, by (2.2), $r=3, s=0$.

Hence, the group $\Gamma^{\prime}$ containing $\Gamma$ as a subgroup of index 2 has a set of canonical generators $\left\{x_{1}, x_{2}, x_{3}, e, c\right\}$ and the restriction map $r: \operatorname{aut}\left(\Gamma^{\prime}, \Gamma\right) \rightarrow \operatorname{aut}(\Gamma): f \mapsto f \mid \Gamma$ is a group isomorphism. However, if $x \in \Gamma^{\prime} \backslash \Gamma$, the inner automorphism

$$
c_{x}: \Gamma^{\prime} \rightarrow \Gamma^{\prime}: \varphi \mapsto x \varphi x^{-1}
$$

is a trivial element in $\bmod \left(\Gamma^{\prime}, \Gamma\right)$, but $r\left(c_{x}\right)$ induces a non-zero element of $\bmod (\Gamma)$. In other words, we have a group epimorphism

$$
\bmod (\Gamma) \rightarrow \bmod \left(\Gamma^{\prime}, \Gamma\right):[f] \mapsto\left[r^{-1}(f)\right],
$$


whose kernel is the group of order 2 generated by the class of $r\left(c_{x}\right)$. Consequently

$$
H(X)=\bmod (\Gamma)=\boldsymbol{Z} / 2 \boldsymbol{Z} \propto \bmod \left(\Gamma^{\prime}, \Gamma\right)
$$

and we are going to see that this is, in fact, a direct product.

We remark that the surface $X$ has no boundary, but since it has genus 3 , then the quotient of $X$ by the hyperelliptic involution has boundary and then we can apply Theorem 4.5. Then

$$
\bmod \left(\Gamma^{\prime}, \Gamma\right)=\left\langle\tau, \delta_{1}, \delta_{2}: \delta_{1} \delta_{2} \delta_{1}=\delta_{2} \delta_{1} \delta_{2} ; \delta_{i} \tau \delta_{i}=\tau, i=1,2 ; \tau^{2}=\left(\delta_{1} \delta_{2}\right)^{3}=1\right\rangle .
$$

In fact $\tau, \delta_{1}$ and $\delta_{2}$ are the classes $\bmod \operatorname{inn}\left(\Gamma^{\prime}\right)$ of the following automorphisms of $\Gamma^{\prime}$ (see (4.2) and (4.4)):

$$
\alpha_{1}:\left\{\begin{array}{l}
x_{1} \mapsto x_{2}, \\
x_{2} \mapsto x_{2} x_{1} x_{2}, \\
x_{3} \mapsto x_{3}, \\
e \mapsto e, \\
c \mapsto c ;
\end{array} \quad \alpha_{2}:\left\{\begin{array}{l}
x_{1} \mapsto x_{1}, \\
x_{2} \mapsto x_{3}, \\
x_{3} \mapsto x_{3} x_{2} x_{3}, \\
e \mapsto e, \\
c \mapsto c ;
\end{array} \quad \widehat{\tau}:\left\{\begin{array}{l}
x_{1} \mapsto x_{1}, \\
x_{2} \mapsto x_{1} x_{2} x_{1}, \\
x_{3} \mapsto x_{1} x_{2} x_{3} x_{2} x_{1}, \\
e \mapsto e^{-1}, \\
c \mapsto e^{-1} c e .
\end{array}\right.\right.\right.
$$

To compute the restrictions $r\left(\alpha_{1}\right), r\left(\alpha_{2}\right)$ and $r(\hat{\tau})$ we must write the generators $z_{i}$ of $\Gamma$ in terms of the ones of $\Gamma^{\prime}$. It is not hard to see that

$$
z_{1}=c x_{1} ; \quad z_{2}=x_{1} c x_{1} x_{2} ; \quad z_{3}=x_{2} x_{1} c x_{1} x_{2} x_{3} .
$$

From this it follows immediately that

$$
z_{2} z_{3}=x_{2} x_{3} ; \quad z_{1} z_{2}=x_{1} x_{2} ; \quad z_{1} z_{2}^{2} z_{3}=x_{1} x_{3} .
$$

These relations are useful to compute the restrictions of $\alpha_{1}, \alpha_{2}$ and $\hat{\tau}$ which are also denoted by $\alpha_{1}, \alpha_{2}$ and $\hat{\tau}$ :

$$
\alpha_{1}:\left\{\begin{array}{l}
z_{1} \mapsto z_{1}^{2} z_{2}, \\
z_{2} \mapsto z_{2}^{-1} z_{1}^{-1} z_{2}, \\
z_{3} \mapsto z_{3} ;
\end{array} \quad \alpha_{2}:\left\{\begin{array}{l}
z_{1} \mapsto z_{1}, \\
z_{2} \mapsto z_{2}^{2} z_{3}, \\
z_{3} \mapsto z_{3}^{-1} z_{2}^{-1} z_{3} ;
\end{array} \quad \hat{\tau}:\left\{\begin{array}{l}
z_{1} \mapsto z_{3}^{-2} z_{2}^{-2} z_{1}^{-1} \\
z_{2} \mapsto z_{1} z_{2}^{2} z_{3}^{2} z_{2}^{-1} z_{1}^{-1} \\
z_{3} \mapsto z_{1} z_{2} z_{3}^{-1} z_{2}^{-1} z_{1}^{-1}
\end{array}\right.\right.\right.
$$

Moreover, $x_{1} \in \Gamma^{\prime} \backslash \Gamma$ and so the automorphism $\hat{h}$ of $\Gamma$ given by 'conjugation by $x_{1}$ ' is defined by

$$
\hat{h}:\left\{\begin{aligned}
z_{1} & \mapsto z_{1}^{-1} \\
z_{2} & \mapsto z_{1} z_{2}^{-1} z_{1}^{-1} \\
z_{3} & \mapsto z_{1} z_{2}^{2} z_{3}^{-1} z_{2}^{-1} z_{1}^{-1}
\end{aligned}\right.
$$

Now, $\hat{h}$ commutes with $\alpha_{2}$ and $\hat{\tau}$. Moreover, if $w=z_{1} z_{2}$, then $\hat{h} \circ \alpha_{1}=C_{w} \circ\left(\alpha_{1} \circ \hat{h}\right)$, where $C_{w}$ denotes 'conjugation by $w$ '. Thus, if $h$ is the class of $\hat{h} \bmod \operatorname{inn}(\Gamma), h$ commutes with $\delta_{1}, \delta_{2}$ and $\tau$, and so

$$
H(X)=(\boldsymbol{Z} / 2 \boldsymbol{Z}=\langle h\rangle) \times \bmod \left(\Gamma^{\prime}, \Gamma\right) .
$$


Notice also that $\delta_{1}, \tau$ and $\delta_{2} h$ generate $\bmod (\Gamma)$, because $\left(\delta_{1} \cdot\left(\delta_{2} h\right)\right)^{3}=h$. Hence, the assignment

$$
\tau \mapsto y ; \quad \delta_{1} \mapsto a ; \quad \delta_{2} \mapsto b
$$

induces an isomorphism between $G(X)$ and $\bmod \left(\Gamma^{\prime}, \Gamma\right)$.

In this way we have reproved Theorem 6.1 , with an explicit representation of a set of generators of $H(X)$, which are not those given by Birman and Chillingworth, as classes of automorphisms of the NEC group $\Gamma$ that uniformizes $X$.

Acknowledgements. The authors are grateful to the referees for their helpful comments and corrections. E.B. and A.F.C. were partly supported by DGICYT PB 98-0017 and J.M.G. was partly supported by DGICYT PB 98-0756.

\section{References}

1. N. L. Alling And N. Greenleaf, Foundations of the theory of Klein surfaces, Lecture Notes in Mathematics, vol. 219 (Springer, Berlin, 1971).

2. J. S. Birman, Braids, links and mapping class groups, Annals of Mathematical Studies, vol. 82 (Princeton University Press, Princeton, NJ, 1975).

3. J. S. Birman and D. R. Chillingworth, On the homeotopy group of a nonorientable surface, Proc. Camb. Phil. Soc. 71 (1972), 437-448.

4. J. S. BiRman AND M. Hilden, On the mapping class groups of closed surfaces as covering spaces, Ann. Math. Stud. 66 (1971), 81-115.

5. E. Bujalance, J. J. Etayo, J. M. Gamboa and G. Gromadzki, Automorphism groups of compact bordered Klein surfaces, Lecture Notes in Mathematics, vol. 1439 (Springer, Berlin, 1990).

6. W. J. Harvey, On branch loci in Teichmüller space, Trans. Am. Math. Soc. 153 (1971), $387-399$.

7. W. J. HaRvey and C. Maclachlan, On mapping class groups and Teichmüller spaces, Proc. Lond. Math. Soc. (3) 30 (1975), 496-512.

8. A. Hatcher and W. Thurston, A presentation for the mapping class group of a closed orientable surface, Topology 19 (1980), 221-237.

9. A. M. MAcBeath, The classification of non-Euclidean plane crystallographic groups, Can. J. Math. 19 (1967), 1192-1205.

10. A. M. Macbeath And D. Singerman, Spaces of subgroups and Teichmüller space, Proc. Lond. Math. Soc. 31 (1975), 211-256.

11. W. Magnus, Braids and Riemann surfaces, Commun. Pure Appl. Math. 25 (1972), 151161.

12. W. Thurston, Three-dimensional geometry and topology, vol. 1 (Princeton University Press, Princeton, NJ, 1997).

13. H. Zieschang, On the homeotopy groups of surfaces, Math. Annln 206 (1973), 1-21. 